\title{
Louse-borne relapsing fever (Borrelia recurrentis) diagnosed in 15 refugees from northeast Africa: epidemiology and preventive control measures, Bavaria, Germany, July to October 2015
}

M Hoch ${ }^{2}$, A Wieser ${ }^{34}$, T Löscher ${ }^{3}$, G Margos ${ }^{1}$, F Pürner ${ }^{2}$, J Zühl 5 , M Seilmaier ${ }^{6}$, L Balzer ${ }^{6}$, W Guggemos ${ }^{6}$, A Rack-Hoch 7 , U von Both ${ }^{7}$, K Hauptvogel ${ }^{8}$, K Schönberger ${ }^{2}$, W Hautmann ${ }^{2}$, A Sing ${ }^{1}$, V Fingerle $^{1}$

1. German National Reference Centre for Borrelia, Bavarian Health and Food Safety Authority (LGL), Oberschleißheim, Germany

2. Task-Force Infectiology and International Health Regulations, Bavarian Health and Food Safety Authority (LGL), Oberschleißheim, Germany

3. Division of Infectious Diseases and Tropical Medicine, University of Munich (LMU), Munich, Germany

4. Max von Pettenkofer Institute for Hygiene and Medical Microbiology, University of Munich (LMU), Munich, Germany

5. City of Munich, Public Health Authority, Munich, Germany

6. Department for Hematology, Oncology, Immunology, Palliative Medicine, Infectiology and Tropical Medicine, Klinikum München-Schwabing, Munich, Germany

7. Division of Paediatric Infectious Diseases, Hauner Children's Hospital, University of Munich (LMU), Munich, Germany

8. RoMed Klinikum Rosenheim, Rosenheim, Germany

Correspondence: Volker Fingerle (Volker.Fingerle@lgl.bayern.de)

Citation style for this article:

Hoch M, Wieser A, Löscher T, Margos G, Pürner F, Zühl Jürgen, Seilmaier M, Balzer L, Guggemos W, Rack-Hoch A, von Both U, Hauptvogel K, Schönberger K,

Hautmann W, Sing A, Fingerle V. Louse-borne relapsing fever (Borrelia recurrentis) diagnosed in 15 refugees from northeast Africa: epidemiology and preventive

control measures, Bavaria, Germany, July to October 2015. Euro Surveill. 2015;20(42):pii=30046. DOI: http://dx.doi.org/10.2807/1560-7917. ES.2015.20.42.30046

Article submitted on 13 October 2015 / accepted on 22 October 2015 / published on 22 October 2015

We report 15 imported louse-borne relapsing fever (LBRF) cases in refugees in Bavaria, Germany. One patient died. Epidemiological findings confirmed that all were young males from the Horn of Africa (12 from Somalia), who had similar migration routes converging in Sudan continuing through Libya and Italy. The majority likely acquired their infection during migration. Healthcare workers should be aware of LBRF in refugees passing through north Africa to ensure correct treatment and preventive measures.

From July 2015 until October 2015, Louse-borne relapsing fever (LBRF) was diagnosed in 15 refugees originating from the Horn of Africa and arriving in Bavaria, southern Germany, presenting with fever. The main travel route included Sudan, Libya and Italy. In this rapid communication, microbiological confirmation, simple first-line epidemiologic assessment and preventive control measures in relation to these cases are described.

\section{Case investigation}

In all cases, a preliminary diagnosis was established by clinicians based on clinical symptoms and visible spirochetes in Giemsa stained blood films The German National Reference Centre (NRC) for Borrelia in Oberschleißheim was contacted directly by clinicians for confirmation. Microbiological confirmation of LBRF from EDTA blood included: Dark field microscopy (Leitz Dialux 20 microscope, Leitz, Germany, objective 40, occular 10) and PCR targeting $16 \mathrm{~S}$ rRNA, flab, and glpQ followed by sequencing [1-3].

Blood samples were used for in vitro cultures in modified Kelly-Pettenkofer (MKP) medium [4]. A case was regarded as confirmed when sequencing revealed $B$. recurrentis.

Because of a potentially high public health relevance, i.e. establishment of local foci of louse-borne transmission in refugee facilities, treating hospitals and spill over to the local population, epidemiological data to establish preventive measures are of utmost importance. Therefore a standardised questionnaire for case investigations and interviews was developed, distributed and evaluated by the Bavarian Task-Force Infectiology and International Health Regulations. Interviews were performed by public health physicians of local public health agencies as part of the official investigation of infectious diseases of public health concern according to the German federal law, and with the help of an interpreter. The interviews were performed in a hospital setting and by taking into consideration potential emotional and psychological trauma of refugees. Anonymised data were used for further analysis. When interviewing was not possible, information was retrieved from medical records, if available. A leaflet informing about clinical presentation, epidemiology, diagnostics as well as preventive control measures, was developed and sent to clinicians and public health authorities to raise general awareness. 


\section{FIGURE 1}

Travel routes of cases of imported louse-borne relapsing fever in refugees from northeast Africa to Bavaria,

Germany, July-September $2015\left(\mathrm{n}=7^{\mathrm{a}}\right)$

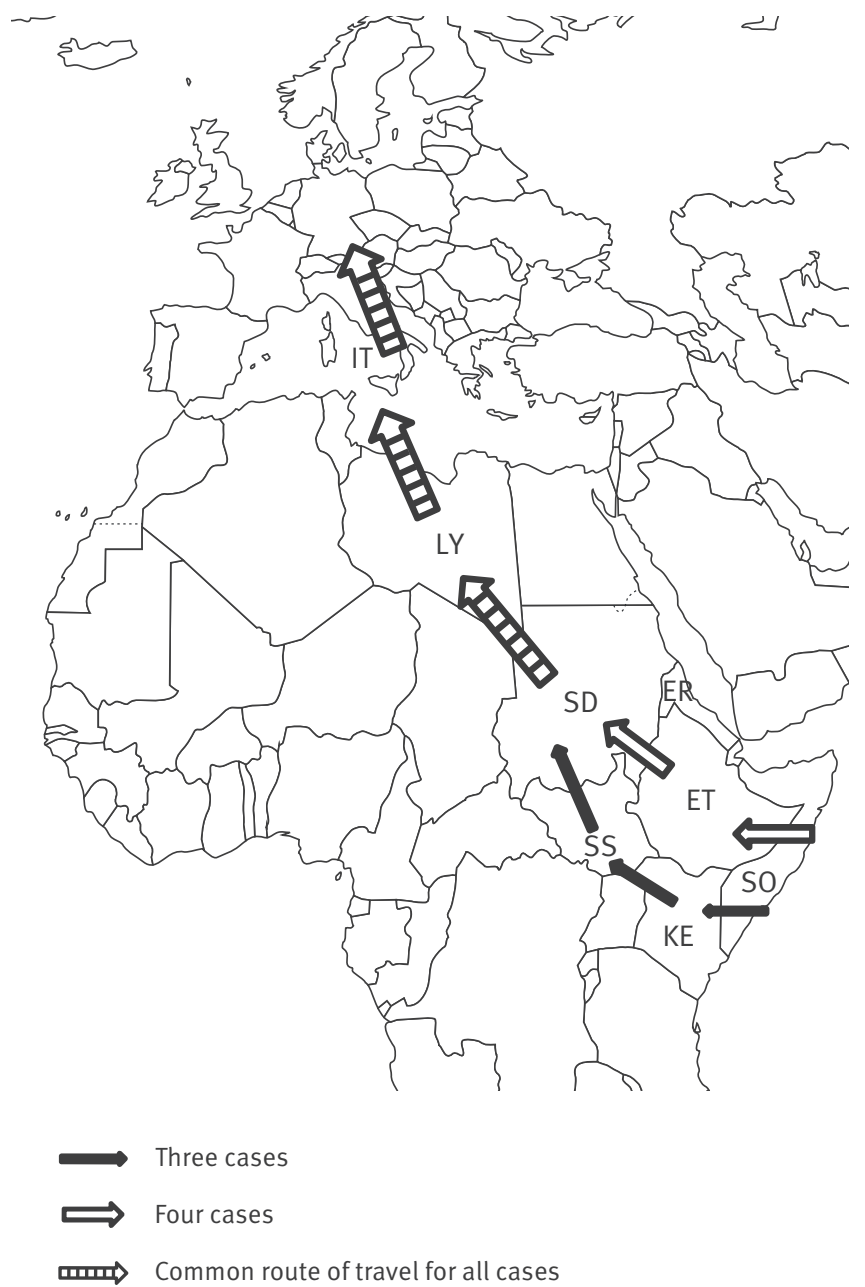

Map obtained from d-maps (http://www.d-maps.com/carte. php?num_car=18215\&lang=en).

${ }^{a}$ Comprehensive information on migration routes was available for seven of the 15 cases.

ER: Eritrea; ET: Ethiopia; IT: Italy; KE: Kenya; LY: Libya; SD: Sudan; SO: Somalia; SS: South Sudan.

\section{Results of the investigations}

Of the 15 suspected LBRF cases submitted to the NRC, 14 were confirmed as being caused by $B$. recurrentis. In one case, spirochetes were visible on Giemsa stained blood films, but insufficient material was provided for molecular diagnosis. However, the epidemiological data suggest LBRF. Details of diagnostic results are shown in Table 1.

Table 2 gives an overview of basic epidemiological information including disease onset and travel history.

All 15 cases were males with a median age of 20 years (range 15 to 33 years) and all originated from northeast

\section{FIGURE 2}

Duration from onset of symptoms to and since arrival in Bavaria for cases of imported louse-borne relapsing fever in refugees from northeast Africa to Bavaria, Germany, July-October $2015(\mathrm{n}=15)$

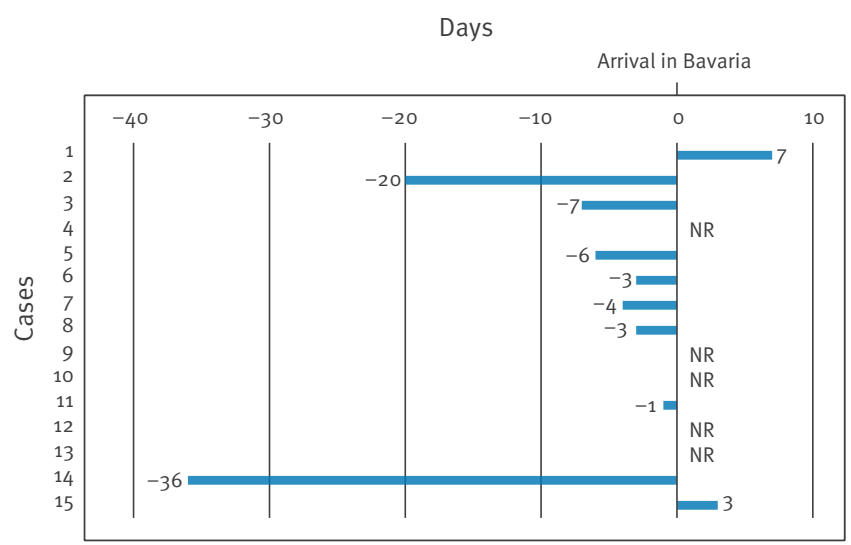

Negative numbers represent an onset of symptoms before arrival in Bavaria whereas positive numbers represent an onset of symptoms after arrival in Bavaria.

NR: not reported.

Africa: 12 from Somalia, two from Eritrea and one from Ethiopia.

Information about migration route was available from 12 patients (Table 2). A comprehensive migration route was indicated by seven patients, all from Somalia: From Somalia via Ethiopia to Sudan $(n=4)$ or via Kenya, South Sudan to Sudan $(n=3)$, all went further to Libya and then via Italy and transit through Austria to Bavaria (Figure 1).

Four patients reported a stay in Italy between two and 14 days, respectively. Six patients reported changing of clothes upon arrival in a refugee camp in Italy. Five patients reported a longer stay in Libya (ranging from 17 days to nine months). Three of them reported to have been exposed to very poor, overcrowded living conditions during transit in Libya. They also reported of fellow refugees with pruritus and infestation with lice. Travel duration of individuals ranged from eight weeks to one year. In eight of the 10 patients with available information, onset of symptoms was before arrival in Munich. Only two reported onset of symptoms after arrival (Figure 2). Nine refugees reported that they had already been accommodated in a refugee facility in Bavaria, four were directly hospitalised upon arrival in Munich, and for two patients information was not specified. Duration of stay in a refugee

Table 3 gives an overview over reported symptoms. All patients were hospitalised and received antibiotic treatment with doxycyline. One of the 15 patients died of multi-organ failure after initiation of antibiotic therapy despite intensive care treatment. Although Jarisch-Herxheimer reaction was not systematically 


\section{TABLE 1}

Diagnostic results of cases of imported louse-borne relapsing fever in refugees from northeast Africa to Bavaria, Germany, July-October 2015 (n=15)

\begin{tabular}{|l|c|c|c|c|c|}
\hline \multirow{2}{*}{ Case } & \multicolumn{2}{|c|}{ Microscopy } & \multicolumn{3}{c|}{ Sequencing } \\
\cline { 2 - 6 } & Darkfield & Giemsa & 16 S rDNA & glpQ & flab \\
\hline $1^{\text {a }}$ & Positive & Positive & Positive & Positive & Positive \\
\hline 2 & Negative & Positive & Positive & Positive & Positive \\
\hline $3^{\text {a }}$ & Positive & Positive & Positive & Positive & Positive \\
\hline $4^{\text {a }}$ & Positive & Positive & Positive & Positive & Positive \\
\hline $5^{\text {a }}$ & Positive & Positive & Positive & Positive & Negative \\
\hline 6 & NA & Positive & NP & NP & NP \\
\hline $7^{\text {a }}$ & Positive & Positive & Positive & Positive & Positive \\
\hline 8 & Negative & Positive & Positive & Negative & Positive \\
\hline 9 & Negative & Positive & Positive & Positive & Negative \\
\hline 10 & Negative & Positive & Positive & IP & Positive \\
\hline 11 & Positive & Positive & Positive & Positive & Positive \\
\hline 12 & Positive & Positive & Positive & Positive & Positive \\
\hline $13^{\text {a }}$ & Positive & Positive & Positive & Positive & Positive \\
\hline 14 & Positive & Positive & Positive & IP & Positive \\
\hline 15 & Positive & Positive & Positive & Positive & Positive \\
\hline
\end{tabular}

a Successful cultivation.

IP: in process; NA: not available; NP: no material provided.

investigated, all 10 patients with available information were reported to have had Jarisch-Herxheimer reaction to antibiotic treatment.

\section{Discussion}

Historically, LBRF was observed as large outbreaks affecting millions of people during wars, civil unrest or under extreme poverty $[5,6]$. Notably, mortality can still be high, above $30 \%$ for untreated cases and $2 \%$ to $6 \%$ for those receiving appropriate treatment [7]. Endemic areas of LBRF were known to exist in northeast Africa in Ethiopia and in neighbouring countries, including Eritrea, Somalia and Southern Sudan, and are suspected in the Peruvian Andes and the Himalayas [5,8-10]. However, according to recent research, it is believed that endemic LBRF hot spots only persist in Ethiopia, occasionally spilling to neighbouring countries $[7,11,12]$.

In Germany, only three cases of imported LBRF were reported since 1999 [13-15]. Imported LBRF among refugees from endemic areas entering the European Union (EU) is not unexpected considering the massive increase in migration of refugees from northeast Africa $[16,17]$. According to official data, Eritrea is one of the main countries of origin of refugees in Bavaria (652 in January-June 2015) [18]. In Germany, 3,284 refugees from Somalia were seeking asylum in 2015 until the month of August [19].

Our data suggest that young male refugees originating from the Horn of Africa may be at particular risk of acquiring LBRF. Accordingly, imported LBRF was found
TABLE 2

Descriptive epidemiological information on cases of imported louse-borne relapsing fever in refugees from northeast Africa to Bavaria, Germany, July-October 2015 $(\mathrm{n}=15)$

\begin{tabular}{|c|c|}
\hline Characteristic & Number of cases \\
\hline \multicolumn{2}{|l|}{ Sex } \\
\hline Male & 15 \\
\hline \multicolumn{2}{|l|}{ Age (years) } \\
\hline $15-20$ & 8 \\
\hline $20-25$ & 4 \\
\hline $25-33$ & 3 \\
\hline \multicolumn{2}{|l|}{ Country of origin } \\
\hline Somalia & 12 \\
\hline Eritrea & 2 \\
\hline Ethiopia & 1 \\
\hline \multicolumn{2}{|l|}{ Migration route } \\
\hline SO-KE-SS-SD-LY-IT & 3 \\
\hline SO-ET-SD-LY-IT & 4 \\
\hline SO-unknown-SD-LY-IT & 1 \\
\hline From SO via LY & 3 \\
\hline From ER via LY and IT & 1 \\
\hline NR & 3 \\
\hline \multicolumn{2}{|l|}{ Travel duration } \\
\hline 1-2 months & 1 \\
\hline $2-5$ months & 6 \\
\hline 5 months -1 year & 1 \\
\hline NR & 7 \\
\hline \multicolumn{2}{|l|}{ Onset of symptoms } \\
\hline Before arrival in Bavaria & 8 \\
\hline After arrival in Bavaria & 2 \\
\hline NR & 5 \\
\hline \multicolumn{2}{|c|}{ Stay in a refugee facility in Bavaria } \\
\hline Yes & 9 \\
\hline No & 4 \\
\hline NR & 2 \\
\hline
\end{tabular}

ER: Eritrea; ET: Ethiopia; IT: Italy; KE: Kenya; LY: Libya; NR: not reported; SD: Sudan; SO: Somalia; SS: South Sudan.

in three young male refugees from Eritrea only recently in Switzerland and the Netherlands [20,21].

Onset of symptoms in the eight of 10 patients with available information in our series was just before or shortly after arrival in Bavaria. None of them reported recurring fever episodes. Hence, we assume an early stage of disease in them. Considering a mean incubation period of four to eight days (range 2 to 15 days) [22] and a duration of migration ranging from eight weeks to one year, infection in these eight patients has most likely been acquired towards the end of their journeys, hence in Libya or Italy. The patients could only provide rough estimates of the exact travel duration and accordingly very limited information on when they entered or left transit countries. Therefore, the exact place of infection remains unclear. However, especially 
Reported symptoms and outcome in louse-borne relapsing fever in refugees from northeast Africa to Bavaria, Germany, July- October 2015

\begin{tabular}{|c|c|c|c|c|c|c|c|c|c|c|}
\hline Case & $\begin{array}{c}\text { Symptoms at the } \\
\text { beginning of the } \\
\text { journey }\end{array}$ & $\begin{array}{l}\text { Fever } \\
>38^{\circ} \mathrm{C}\end{array}$ & $\begin{array}{l}\text { Recurring } \\
\text { fever }\end{array}$ & $\begin{array}{l}\text { Severe } \\
\text { malaise }\end{array}$ & $\begin{array}{l}\text { Headache } \\
\text { Myalgia }\end{array}$ & $\begin{array}{c}\text { Other } \\
\text { symptoms }\end{array}$ & $\begin{array}{l}\text { Infestation } \\
\text { with lice }\end{array}$ & $\begin{array}{c}\text { Pruritus/ } \\
\text { skin lesions }\end{array}$ & Hospitalisation & Recovery \\
\hline 1 & No & No & No & NR & NR & No & No & NR & Yes & Yes \\
\hline 2 & NR & Yes & NR & NR & NR & NR & NR & NR & Yes $^{f}$ & NR \\
\hline 3 & NR & Yes & NR & NR & NR & NR & NR & NR & Yes $^{f}$ & NR \\
\hline 4 & No & Yes & $\begin{array}{c}\text { Several } \\
\text { episodes }\end{array}$ & NR & No & Yes $^{\mathrm{a}}$ & Yes $^{b}$ & No & ICU & Yes \\
\hline 5 & No & Yes & No & Yes & Yes & Yes $^{c}$ & No & Yes & Yes & Yes \\
\hline 6 & No & Yes & No & Yes & Yes & No & No & No & Yes & Yes \\
\hline 7 & No & Yes & No & Yes & Yes & No & Yes & No & Yes & Yes \\
\hline 8 & No & Yes & No & Yes & Yes & No & NR & No & Yes & Yes \\
\hline 9 & NR & Yes & NR & NR & NR & NR & NR & NR & Yes $^{f}$ & NR \\
\hline 10 & NR & Yes & NR & NR & NR & $\mathrm{NR}$ & NR & NR & Yes $^{f}$ & NR \\
\hline 11 & NR & Yes & NR & Yes & NR & Yes $^{c, d}$ & Yes & Yes & Yes & Yes \\
\hline 12 & NR & Yes & NR & Yes & NR & No & NR & Yes & Yes & Yes \\
\hline 13 & NR & Yes & NR & NR & NR & NR & NR & NR & Yes & $\mathrm{No}^{\mathrm{e}}$ \\
\hline 14 & NR & Yes & No & Yes & yes & No & Yes & No & Yes & Yes \\
\hline 15 & NR & Yes & No & Yes & yes & Yes $^{c}$ & No & Yes & Yes & Yes \\
\hline
\end{tabular}

ICU: intensive care unit; NR: not reported.

abdominal pain.

${ }^{b}$ Infestation was not reported, but seen at hospital admission.

c Jaundice.

${ }^{\mathrm{d}}$ Vomiting.

e Patient died.

${ }^{f}$ Lost to follow-up.

for five cases who reported onset of symptoms within days before or shortly after arrival, an infection not only in Libya but also in Italy or while crossing the Mediterranean Sea is possible. Two patients reported an onset of symptoms 20 and 36 days, respectively, before arrival in Germany. The patient with the longer duration of symptoms reported that he had travelled through Ethiopia. For the two patients and those with insufficient information regarding the travel route, an infection while travelling through the known endemic hot spot Ethiopia or Sudan could not be ruled out. However, considering incubation time, onset of symptoms, travel duration and the fact that six patients did report no symptoms when leaving their country of origin, we assume that LBRF was acquired later, at places along migration routes with established endemic LBRF foci.

We are aware that the data retrieved during interviews are limited by recall bias and language problems. In particular, the lack of exact data on duration of the journeys remains problematic when estimating the probable place of infection. Indeed, we conclude that LBRF has already been introduced in places along migration routes, most likely in Libya, on refugee boats crossing the Mediterranean Sea and perhaps in Italy. Looking at specified migration routes, the introduction of LBRF to these places is possible by refugees who acquired the disease in Ethiopia.

Interestingly, not a single LBRF infection was found in refugees originating from other African regions though their migration routes partially overlap [23].

The current assessment provides no evidence that transmission occurred while the individuals were in Bavaria. However, public health measures were implemented to prevent further transmission, since nine of the 15 refugees already stayed in a refugee facility in Bavaria before they were diagnosed. As primary preventive public health measure, basic hygiene was recommended which includes changing, washing and drying of clothes and bedding at $\geq 60^{\circ} \mathrm{C}$ on a regular basis for infected individuals and close contacts [24]. Additionally, active contact tracing of cases in terms of symptoms, infestation with body lice and assessment of living conditions was recommended as a further precaution. Furthermore, it was recommended to actively offer washing of clothes upon arrival, especially, but not limited to refugees from endemic areas. As the detection of infestation with lice might generally not be very reliable (in our assessment only reported in four patients) these preventive measures have become pivotal $[22,24]$. 
To raise awareness in the medical and public health communities, public health authorities and physicians throughout Bavaria have been informed about clinical symptoms and general epidemiology of LBRF. Leaflets describing LBRF and recommended public health measures were distributed nationally. Public health institutions in Libya and Italy were already informed by the Robert-Koch-Institute (RKI) (personal communication, Dr H Wilking, RKI, September 2015) as well as further organisations such as the World Health Organization (WHO) and Médecins Sans Frontières (MSF).

\section{Conclusions}

Young male refugees originating from the Horn of Africa seem to be at major risk for acquiring LBRF. LBRF most likely was not acquired in their countries of origin but on migration routes, most likely in Libya or Italy. No evidence for transmission of LBRF infections in Bavaria was found. However meaningful preventive control measures were implemented. The findings of our investigation should be strengthened by further epidemiological studies and followed by a pan-European prevention strategy.

\section{Conflict of interest}

None declared.

\section{Authors' contributions}

Wrote the manuscript: MH, VF, AR; performed epidemiological analysis: $M H, A R, F P$; interviewed the patients: JZ; performed laboratory investigation: GM, VF, AW; revised the manuscript: KS, WH, TL, AS, GM; cared for the patients: MS, LB, UB, WG, KH.

\section{References}

1. SafdieG, FarrahıY, YahiaR, MarvaE, WilamowskiA, SawalhaSS, et al. Molecular characterization of Borrelia persica, the agent of tick borne relapsing fever in Israel and the Palestinian Authority. PLOS ONE. 2010;5(11):e14105. DOI: 10.1371/journal. pone.0014105 PMID: 21124792

2. VenczelR, KnokeL, PavlovicM, DzaferovicE, VaculovaT, SilaghiC, et al. A novel duplex real-time PCR permits simultaneous detection and differentiation of Borrelia miyamotoi and Borrelia burgdorferi sensu lato. Infection. 2015;14:14.PMID: 26168860

3. RadulovićŽ, MilutinovićM, TomanovićS, MulengaA. Detection of Borrelia-specific 16S rRNA sequence in total RNA extracted from Ixodes ricinus ticks. Arq Bras Med Vet Zootec.2010;62(4):862-7.

4. MargosG, StockmeierS, Hizo-TeufelC, HepnerS, FishD, DautelH, et al. Long-term in vitro cultivation of Borrelia miyamotoi. Ticks Tick Borne Dis. 2015;6(2):181-4. DOI: 10.1016/j. ttbdis.2014.12.001 PMID: 25561082

5. RaoultD, RouxV. The body louse as a vector of reemerging human diseases.Clin Infect Dis. 1999;29(4):888-911. DOI: 10.1086/520454 PMID: 10589908

6. CutlerSJ. Possibilities for relapsing fever reemergence.Emerg Infect Dis. 2006;12(3):369-74. DOI: 10.3201/eid1203.050899 PMID: 16704771

7. CutlerSJ, AbdissaA, TrapeJF. New concepts for the old challenge of African relapsing fever borreliosis.Clin Microbiol Infect. 2009;15(5):400-6. DOI: 10.1111/j.1469-0691.2009.02819.x PMID: 19489922

8. PorcellaSF, RaffelSJ, SchrumpfME, SchrieferME, DennisDT, SchwanTG. Serodiagnosis of Louse-Borne relapsing fever with glycerophosphodiester phosphodiesterase (GlpQ) from
Borrelia recurrentis.J Clin Microbiol. 2000;38(10):3561-71.PMID: 11015364

9. de JongJ, WilkinsonRJ, SchaeffersP, SondorpHE, DavidsonRN. Louse-borne relapsing fever in southern Sudan.Trans R Soc Trop Med Hyg. 1995;89(6):621. DOI: 10.1016/00359203(95)90414-X PMID: 8594674

10. RaoultD, BirtlesRJ, MontoyaM, PerezE, Tissot-DupontH, RouxV, et al. Survey of three bacterial louse-associated diseases among rural Andean communities in Peru: prevalence of epidemic typhus, trench fever, and relapsing fever. Clin Infect Dis. 1999;29(2):434-6. DOI: 10.1086/520229 PMID: 10476755

11. YimerM, MuluW, AyalewW, AberaB. Louse-borne relapsing fever profile at Felegehiwot referral hospital, Bahir Dar city, Ethiopia: a retrospective study.BMC Res Notes. 2014;7(250):250. DOI: 10.1186/1756-0500-7-250 PMID: 24742342

12. CutlerSJ. Relapsing fever--a forgotten disease revealed. Appl Microbiol. 2010;108(4):1115-22. DOI: 10.1111/j.13652672.2009.04598.x PMID: 19886891

13. Robert Koch Institute (RKI),. Rueckfallfieber - selten, aber ernst zu nehmen [Relapsing fever - rare, but to be taken seriously]. Berlin: RKI. 6 Oct 2015. Epidemiologisches Bulletin 44/2000. German. Available from: http://edoc.rki.de/documents/rki_fv/ re4mThxdAoes/PDF/22dqy6pQ75wg.pdf.

14. Robert Koch Institute (RKI). Infektionsepidemiologisches Jahrbuch meldepflichtiger Krankheiten fuer 2001 [Yearbook of notifiable infectious diseases in Germany, 2001]. Berlin: RKI. 9 Oct 2015. German. Available from: http:// www.rki.de/DE/Content/Infekt/Jahrbuch/Jahrbuch_2001. pdf?__blob=publicationFile.

15. Robert Koch Institute (RKI). Infektionsepidemiologisches Jahrbuch meldepflichtiger Krankheiten fuer 2014. [Yearbook of notifiable infectious diseases in Germany, 2014] Berlin: RKI. 9 Oct 2015. Available from: http://www.rki.de/DE/Content/ Infekt/Jahrbuch/Jahrbuch_2014.pdf?__blob=publicationFile.

16. United Nations High Commissioner for Refugees (UNHCR). Sharp increase in number of Eritrean refugees and asylumseekers in Europe, Ethiopia and Sudan. Geneva: UNHCR. 31 Aug 2015. Available from: http://www.unhcr.org/5465fea1381.html.

17. European Centre for Disease Prevention and Control (ECDC). Louse-borne relapsing fever in the Netherlands. Stockholm: ECDC. 6 Oct 2015. Available from: http://ecdc.europa.eu/ en/publications/Publications/louse-borne-relapsing-fevernetherlands-rapid-risk-assessment.pdf.

18. Asylsozialpolitik StMAS. Daten und Fakten. [Social policy with respect to asylum issues. Numbers and facts. Bavarian State Ministry of Labour and Social Welfare, Family Affairs and Integration] Munich: Bayerisches Staatsministerium fuer Arbeit und Soziales (StMAS); 2015 [06/10/2015]; Available from: http://www.zukunftsministerium.bayern.de/migration/asyl/ index.php.

19. United Nations High Commissioner for Refugees (UNHCR). UNHCR Population Statistics Database. Geneva: UNHCR. 6 Oct 2015. Available from: http://popstats.unhcr.org/en/ overview\#_ga=1.191407852.716888311.1444119362.

20. WiltingKR, StienstraY, SinhaB, BraksM, CornishD, GrundmannH. Louse-borne relapsing fever (Borrelia recurrentis) in asylum seekers from Eritrea, the Netherlands, July 2015. Euro Surveill. 2015;20(30):21196. DOI: 10.2807/15607917.ES2015.20.30.21196 PMID: 26250069

21. GoldenbergerD, ClaasGJ, Bloch-InfangerC, BreidthardtT, SuterB, MartinezM, et al. Louse-borne relapsing fever (Borrelia recurrentis) in an Eritrean refugee arriving in Switzerland, August 2015. Euro Surveill. 2015;20(32):21204. DOI: $10.2807 / 1560-7917$. ES2015.20.32.21204 PMID: 26290486

22. European Centre for Disease Prevention and Control (ECDC). Louse-borne relapsing fever; Factsheet for health professionals. Stockholm: ECDC; 6 Oct 2015. Available from: http://ecdc.europa.eu/en/healthtopics/emerging_and_vectorborne_diseases/vector-borne_diseases/louse-bornerelapsingfever/Pages/Factsheet-for-health-professionals.aspx.

23. Interactive map of migration. 2014 MTM Map on Mixed Migration Routes in the MTM Region [Internet]. 2014. [31/o8/2015]; Available from: http://www.imap-migration.org/ index.php?id $=470$

24. Centers for Disease Control and Prevention (CDC). Body lice - Frequently asked questions. Atlanta: CDC; 2013. Available from: http://www.cdc.gov/parasites/lice/body/gen_info/faqs. html. 